\title{
Overwhelming Post-Splenectomy Infection Syndrome: Variability in Timing With Similar Presentation
}

\author{
Anupam K. Gupta ${ }^{1}$, Oscar A. Vazquez ${ }^{2}$ \\ 1. Minimally Invasive Surgery, University of Miami Hospital, Miami, USA 2. Surgery, Charles E. Schmidt College of \\ Medicine, Florida Atlantic University, Boca Raton, USA
}

Corresponding author: Anupam K. Gupta, dranupamkumargupta@gmail.com

\begin{abstract}
Overwhelming post-splenectomy infection (OPSI) syndrome is a rare and well-known entity that rapidly progresses with poor outcomes. Two patients underwent splenectomy after trauma and later presented with flu-like symptoms and thrombocytopenia, which then progressed to fulminant sepsis and death. The first patient had sepsis 20 days post-splenectomy, and the second patient underwent splenectomy 15 years before presentation. Both patients expired within 24 hours of the onset of symptoms. Even with no specific criteria for diagnosis, prompt identification of the overwhelming post-splenectomy infection is necessary; however, the prognosis is usually poor, even with aggressive treatment.
\end{abstract}

Categories: Allergy/Immunology, General Surgery, Infectious Disease Keywords: opsi, splenectomy, infection, sepsis

\section{Introduction}

Splenectomy is performed as a life-saving surgery in the trauma setting as the spleen is the most commonly injured organ in blunt abdominal trauma (up to $31 \%-50 \%$ of cases) [1,2]. There has been a decrease in the number of splenectomy patients using angioembolization, but a subset of patients who fail angioembolization still need a splenectomy if bleeding is present from secondary splenic rupture [3,4]. Overwhelming post-splenectomy infection (OPSI) syndrome is a well-known entity that is rare and fatal due to its rapidly progressive and fulminant course over 12-24 hours after the first sign of "flu-like" symptoms $[5,6]$. Although the first instances of bacterial sepsis occurring post-splenectomy were first documented in 1952 by King and Shumacker in infants and children, it is known to affect asplenic adults $[7,8]$.

\section{Case Presentation}

\section{Case \#1}

Received 07/28/2020

Review began 08/03/2020 Review ended 08/15/2020 Published 08/21/2020

๑) Copyright 2020 Gupta et al. This is an open access article distributed under the terms of the Creative Commons Attribution License CC-BY 4.0., which permits unrestricted use, distribution, and reproduction in any medium, provided the original author and source are credited.
An 80-year-old male patient with a past medical history of a myeloproliferative disorder, coronary artery disease, and diabetes presented to the emergency room after a motor vehicle collision. On arrival, the patient's vital signs were significant for tachycardia of 115 beats per minute and blood pressure of $100 \mathrm{~mm}$ $\mathrm{Hg}$ systolic. The primary survey was unremarkable, and a secondary survey showed bruises and abrasions on the left side of the abdomen. A bedside focused ultrasonography for assessment of trauma (FAST) exam was positive for free fluid in the abdomen. The patient's vital signs stabilized with a one-liter bolus of Ringer's lactate solution. The patient then underwent a CT scan of the abdomen and pelvis, which showed splenomegaly and a splenic laceration (Figure 1). The patient underwent immediate angioembolization for splenic bleed. The patient was monitored for trends in hemoglobin, which showed a gradual decline with increasing abdominal pain, and this prompted the team to perform a splenectomy with a washout of blood clots. The postoperative course was uneventful, with the patient ambulating and tolerating a regular diet. Surgical staples were removed on day fourteen, and he was vaccinated for Hemophilus influenza, Streptococcal pneumonia, and Neisseria meningitides. The patient was scheduled to be transferred to a rehab facility when he complained of non-specific symptoms of body aches and nasal congestion. The clinical exam was remarkable only for a low-grade fever of 100.5 degrees Fahrenheit. Blood work significant values were thrombocytopenia of $20,000 / \mathrm{mL}$ (the normal value is $150,000-400,000 / \mathrm{mL}$ ) and white blood cell count of $7,000 / \mathrm{mL}$ (the normal value being 4,500 to $11,000 \mathrm{WBC} / \mathrm{mL}$ ). The patient was initiated on broad-spectrum antibiotics because of fever, and cultures were drawn from blood, sputum, and urine. A chest x-ray and CT scan of the abdomen and pelvis revealed no foci of infection or fluid collection around the operative site. Over the next few hours, the patient showed increasing tachycardia and a drop in his mean arterial pressure to $60 \mathrm{mmHg}$ from $80 \mathrm{mmHg}$. The patient was then monitored in the intensive care unit for vasopressor support with a sharp decline in clinical status. The patient became tachycardic to 120 beats per minute sinus rhythm, tachypneic at 20 breaths per minute, and hypotensive. The patient was intubated, supported with increasing vasopressors and was given steroids and platelets. The patient then progressed in acidosis and needed multiple cycles of cardiopulmonary resuscitation. The patient expired within 20 hours from the identification of thrombocytopenia. Blood cultures showed evidence of Klebsiella 


\section{Cureus}

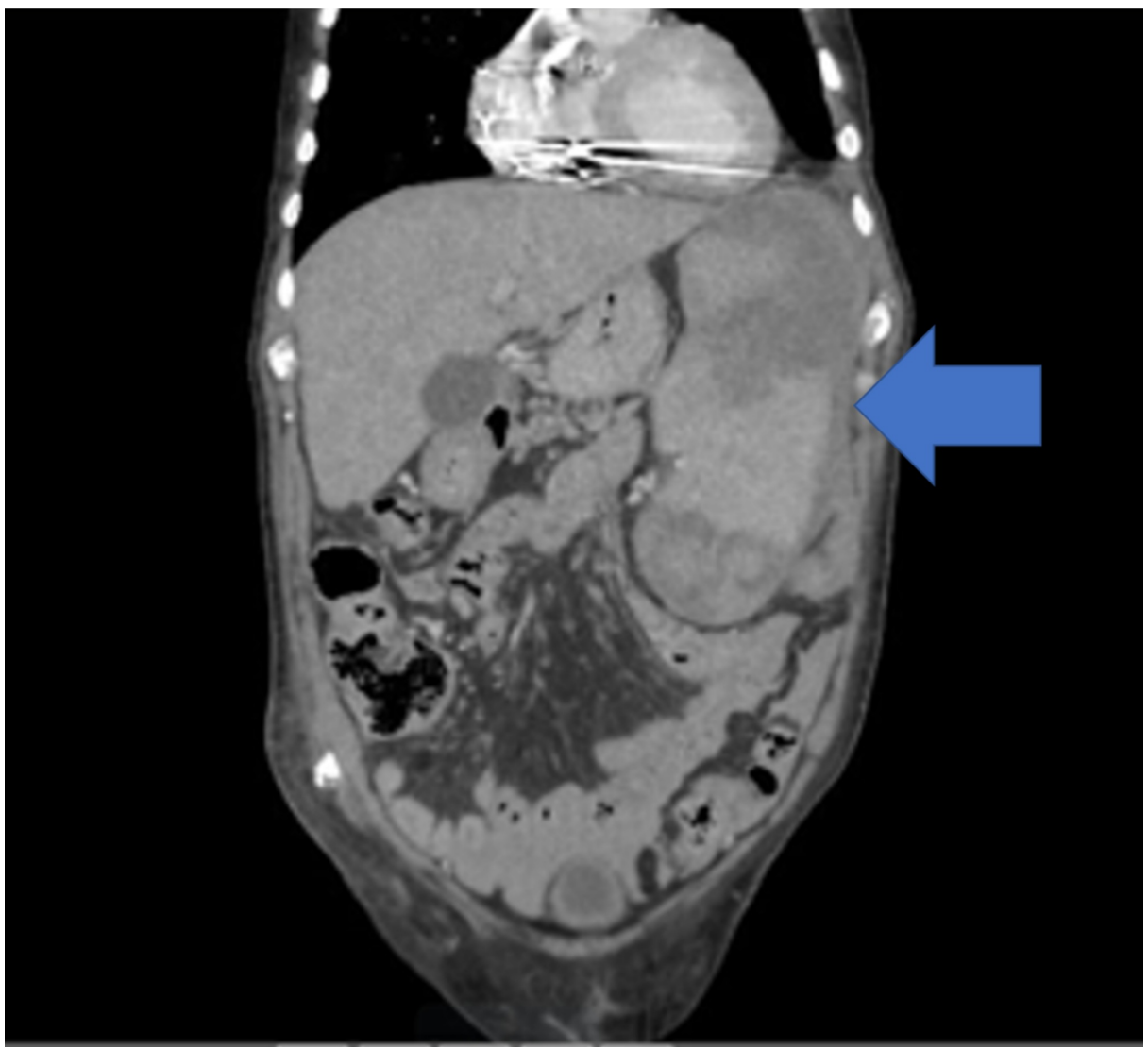

\section{FIGURE 1: Computed tomography scan showing splenomegaly and splenic laceration}

\section{Case \#2}

A 54-year-old female patient with a past medical history of Hepatitis C infection, diabetes mellitus, hyperlipidemia, and hypertension presented with non-specific symptoms of mild weakness, lethargy, and development of rashes which began the day before. Her surgical history was remarkable for splenectomy 15 years prior due to trauma sustained in a motor vehicle collision. She was alert and conscious; however, vital signs showed tachycardia to 110 beats per minute, tachypnea of 35 breaths per minute, and systolic blood pressure of $100 \mathrm{mmHg}$, which responded after a fluid bolus to $115 / 74 \mathrm{mmHg}$. A detailed clinical exam was significant for rashes over her body (Figures 2, 3). Her blood work was remarkable for a white blood cell count of 33000/mL, thrombocytopenia of 20000/mL, INR 2.2 (normal being 1.1 or below), PTT 56 seconds (normal value are typically 25 to 35 seconds), fibrinogen $67 \mathrm{~g} / \mathrm{L}$ (normal range is 2.0 to $4.0 \mathrm{~g} / \mathrm{L}$ ), creatine 2.3 $\mathrm{mg} / \mathrm{dL}$ (normal range for creatinine in the blood is 0.84 to $1.21 \mathrm{mg} / \mathrm{dL}$ ) and lactic acidosis of $11 \mathrm{mmol} / \mathrm{L}$ (normal blood lactate concentration in unstressed patients is $0.5-1 \mathrm{mmol} / \mathrm{L}$ ). Patients' arterial blood gas analysis showed metabolic and respiratory acidosis with a pH of 7.29, bicarbonate of $17 \mathrm{mEq} / \mathrm{L}, \mathrm{PaCO} 2$ of 70 $\mathrm{mmHg}$, and a PaO2 of $80 \mathrm{mmHg}$. The patient was intubated and underwent a CT scan of the head, chest, abdomen, and pelvis, which revealed pneumatosis in the portal vein for which she underwent an emergent exploratory laparotomy. No pathological finding was seen on exploratory laparotomy, and the patient was immediately transferred with an open abdomen for further resuscitation in the intensive care unit. The patient was continued on blood products and close hemodynamic monitoring with a progressive increase in vasopressor requirements and acidosis over the next few hours. The patient expired over the next few hours despite multiple resuscitation efforts, and her blood, urine, and sputum cultures were unrevealing. 


\section{Cureus}

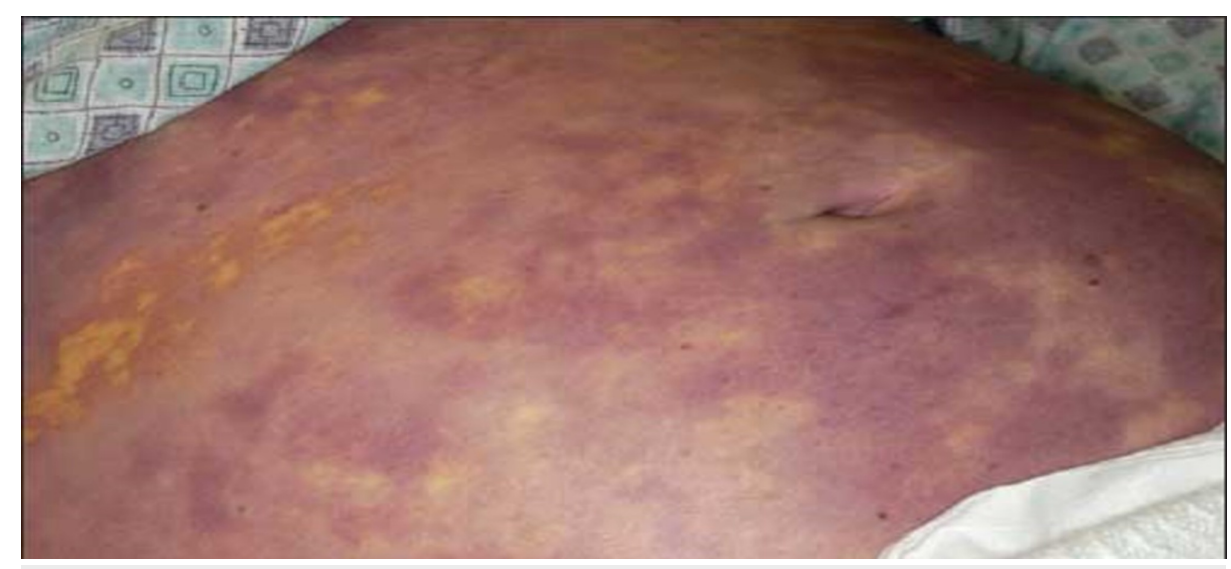

FIGURE 2: Rashes on patient's abdomen

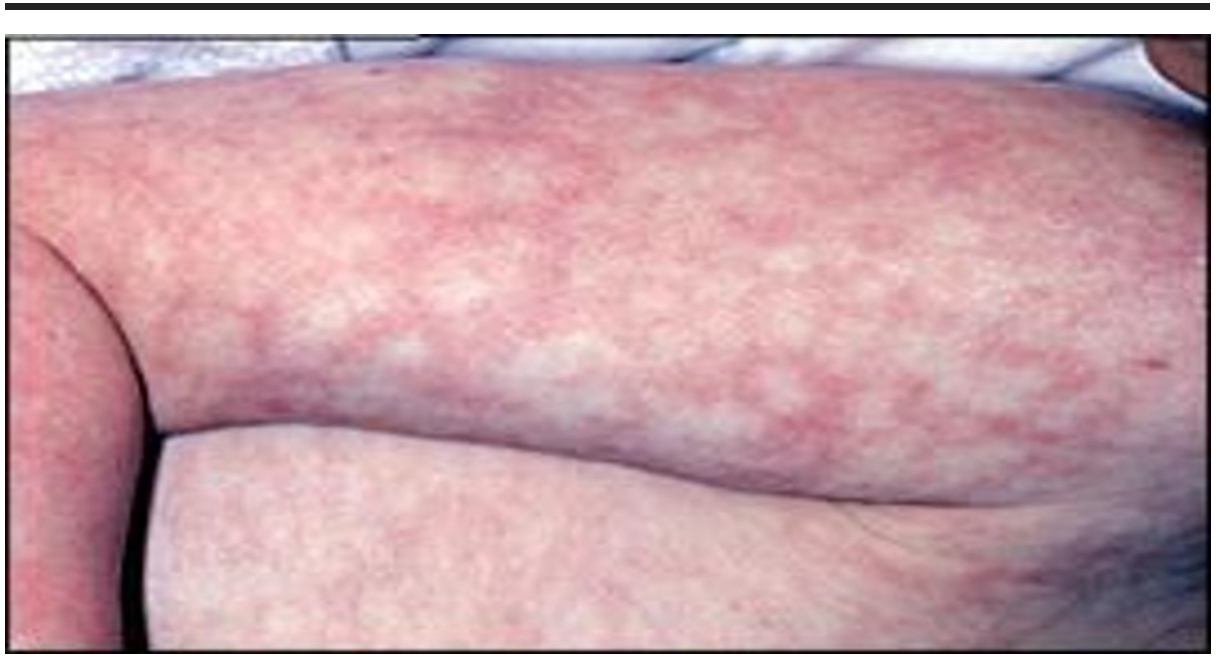

FIGURE 3: Rashes on patient's right arm

\section{Discussion}

OPSI syndrome has a mortality rate of up to $50 \%$, with a prevalence of $0.1 \%-0.5 \%$ [9]. Though the likelihood of this condition manifesting is most likely within three years from splenectomy, there have been reported cases up to 20 years from splenectomy, five years after the second patient presented had their spleen removed [7]. Common pathogens include Streptococcus pneumoniae, Haemophilus influenzae type b, group B Streptococcus, Staphylococcus aureus, Salmonella species, Escherichia coli, and other coliforms, Capnocytophaga canimorsus, and Pseudomonas aeruginosa [10]. OPSI syndrome classically begins with "flu-like symptoms" described as a prodrome composed of fever, chills, myalgia, headache, vomiting, and abdominal pain which then rapidly progresses to coma, acidosis, respiratory distress, septic shock, and disseminated intravascular coagulation (DIC) within 24-48 hours [11-13]. It is theorized that the cause of DIC and thrombocytopenia in patients infected with Streptococcus pnemoniae comes from polysaccharidespecific antibodies that activate the complement pathway and promote the deposition of complement fragments directly on to the bacterial capsule to cause thrombotic occlusion [14]. Other theories behind the pathogenesis of OPSI and its fulminant course include the loss of splenic phagocytic function, suppression of lymphocyte sensitivity, and the decreasing serum immunoglobulin levels causing a change in the opsonin system [15]. In later stages, the presentation is similar to that of Waterhouse-Friderichsen syndrome (WFS) with petechial rash and neurological manifestations with autopsy reports indicating the finding of bilateral adrenal hemorrhage [16,17].

Though OPSI syndrome is challenging to diagnose due to a lack of clear diagnostic criteria, clinicians should be extra vigilant in patients with a history of splenectomy presenting with fever, chills, diarrhea, and vomiting. Furthermore, in patients with signs of sepsis or septic shock, at least two sets of blood cultures should be drawn immediately before prompt administration of empiric, broad-spectrum antibiotics. Recommended laboratory investigations in these cases include a complete metabolic panel, liver function tests, blood glucose level, and serum lactate concentration. It is also recommended to draw a peripheral blood smear for the presence of bacteria and Howell-Jolly bodies as a result of asplenia [18]. Kumar et al. 
found that immediate administration of antibiotics increases survival rates and also recommend pneumococcal, Haemophilus influenza type b (Hib), meningococcal, and annual influenza vaccinations in asplenic individuals [19]. Treatment of OPSI is generally aggressive with intravenous fluids, antibiotics, vasopressors, steroids, heparin, packed red blood cells, platelets, cryoprecipitates, and fresh frozen plasma [15]. Due to its fulminant and fatal nature, prevention is of utmost importance in immunocompromised asplenic patients [5]. Recommended broad-spectrum antibiotics for asplenic patients include amoxicillinclavulanic acid, trimethoprim-sulfamethoxazole (TMP/SMX), and cefuroxime since penicillins are now lesseffective against increasing bacterial resistance [20]. Our patient's splenectomies were less than three weeks to fifteen years before presentation, yet they presented similarly with "flu-like" symptoms and thrombocytopenia. The first patient had a positive culture for encapsulated gram-negative organisms, and the second patient had a rash consistent with WFS. Together, they are an example of how rapidly this disease progresses and the variability in timing regarding the presentation post-splenectomy.

\section{Conclusions}

OPSI syndrome may progress quickly, leading to fatal septic shock. The initial presentation can be nonspecific flu-like symptoms with blood work significant for thrombocytopenia. It may also be caused by encapsulated gram-negative organisms like Escherichia coli and Klebsiella species, and, at times, there is no evidence of source on imaging and cultures. Finally, OPSI syndrome can occur in the immediate postoperative period to many years later.

\section{Additional Information \\ Disclosures}

Human subjects: Consent was obtained by all participants in this study. Conflicts of interest: In compliance with the ICMJE uniform disclosure form, all authors declare the following: Payment/services info: All authors have declared that no financial support was received from any organization for the submitted work. Financial relationships: All authors have declared that they have no financial relationships at present or within the previous three years with any organizations that might have an interest in the submitted work. Other relationships: All authors have declared that there are no other relationships or activities that could appear to have influenced the submitted work.

\section{References}

1. El-Matbouly M, Jabbour G, El-Menyar A, et al.: Blunt splenic trauma: assessment, management and outcomes. Surgeon. 2016, 14:52-58. 10.1016/j.surge.2015.08.001

2. Martin G, Slim K: Extreme emergency splenectomy. J Visc Surg. 2014, 151:41-44. 10.1016/j.jviscsurg.2013.12.010

3. Imbrogno BF, Ray CE: Splenic artery embolization in blunt trauma. Semin Intervent Radiol. 2012, 29:147149. $10.1055 / \mathrm{s}-0032-1312577$

4. Peitzman AB, Heil B, Rivera L, et al.: Blunt splenic injury in adults: multi-institutional study of the eastern association for the surgery of trauma. J Trauma. 2000, 49:177-189. 10.1097/00005373-200008000-00002

5. Okabayashi T, Hanazaki K: Overwhelming postsplenectomy infection syndrome in adults - a clinically preventable disease. World J Gastroenterol. 2008, 14:176-179. 10.3748/wjg.14.176

6. Francke EL, Neu HC: Post splenectomy infection. Surg Clin North Am. 1981, 61:135-155. 10.1016/s00396109(16)42339-x

7. King H, Shumacker HB Jr: Splenic studies. I. Susceptibility to infection after splenectomy performed in infancy. Ann Surg. 1952, 136:239-242. 10.1097/00000658-195208000-00006

8. Kristinsson SY, Gridley G, Hoover RN, Check D, Landgren O: Long-term risks after splenectomy among 8,149 cancer-free American veterans: a cohort study with up to 27 years follow-up. Haematologica. 2014, 99:392398. 10.3324/haematol.2013.092460

9. Sarangi J, Coleby M, Trivella M, Reilly S: Prevention of post splenectomy sepsis: a population based approach. J Public Health Med. 1997, 19:208-212. 10.1093/oxfordjournals.pubmed.a024611

10. Davidson RN, Wall RA: Prevention and management of infections in patients without a spleen . Clin Microbiol Infect. 2001, 7:657-660. 10.1046/j.1198-743x.2001.00355.x

11. Gopal V, Bisno AL: Fulminant pneumococcal infections in "normal” asplenic hosts. Arch Intern Med. 1977, 137:1526-1530. 10.1001/archinte.1977.03630230022009

12. Chaikof EL, McCabe CJ: Fatal overwhelming postsplenectomy infection. Am J Surg. 1985, 149:534-539. 10.1016/S0002-9610(85)80052-0

13. Leonard AS, Giebink GS, Baesl TJ, Krivit W: The overwhelming postsplenectomy sepsis problem. World J Surg. 1980, 4:423-427. 10.1007/BF02393164

14. Saeland E, Vidarsson G, Leusen JHW, et al.: Central role of complement in passive protection by human IgG1 and IgG2 anti-pneumococcal antibodies in mice. J Immunol. 2003, 170:6158-6164. 10.4049/jimmunol.170.12.6158

15. Machesky KK, Cushing RD: Overwhelming postsplenectomy infection in a patient with penicillin-resistant Streptococcus pneumoniae. Arch Fam Med. 1998, 7:178-180. 10.1001/archfami.7.2.178

16. Guidelines for the prevention and treatment of infection in patients with an absent or dysfunctional spleen . Working Party of the British Committee for Standards in Haematology Clinical Haematology Task Force. BMJ. 1996, 312:430-434.

17. Bouros D, Nicholson AC, Polychronopoulos V, du Bois RM: Acute interstitial pneumonia . Eur Respir J. 2000, 15:412-418. 10.1034/j.1399-3003.2000.15b31.x 


\section{Cureus}

18. Dellinger RP, Levy MM, Rhodes A, et al.: Surviving sepsis campaign: international guidelines for management of severe sepsis and septic shock, 2012. Intensive Care Med. 2012, 39:165-228. 10.1007/s00134-012-2769-8

19. Kumar A, Roberts D, Wood KE, et al.: Duration of hypotension before initiation of effective antimicrobial therapy is the critical determinant of survival in human septic shock. Crit Care Med. 2006, 34:1589-1596. 10.1097/01.CCM.0000217961.75225.E9

20. Brigden ML: Detection, education and management of the asplenic or hyposplenic patient . Am Fam Physician. 2001, 63:499-508. 